\title{
EI Impacto del COVID 19 en la Incidencia de compras en Línea de los Millenials
}

\section{The Impact of COVID-19 in the Online Shopping Incidence of Millenials}

\author{
Maria del Carmen Catache Mendoza, Gloria Pedroza Cantú, Zita Mirthala Huerta Cerda, Ruth Isela \\ Martínez Valdez \\ 1,2,3,4 Universidad Autónoma de Nuevo León, Facultad de Ciencias Químicas, Nuevo León, México. \\ *maría.catachemnd@uanl.edu.mx \\ DOI: https://doi.org/10.29105/revig1.1-6
}

\begin{abstract}
The COVID-19 contingency declaration occurred in March of this year brought with it changes in the buying habits of consumers since the closure of shops, department stores and the reduction of face-to-face attention to the public in banks and other services caused an increase in the number of people making purchases through e-commerce.

This study analyzes how COVID-19 has an impact on the incidence of online shopping among young Millenials. An online survey was applied to 187 people from the Northeast of Nuevo León belonging to the aforementioned generation, in an age range of 25 to 35 years. The results obtained in this study show marked differences between online shopping habits between men and women, who carry out transactions in banking services applications and store pages to make purchases of various products, this to safeguard their health.
\end{abstract}

Key Words: e-commerce, COVID-19, Millennial generation

\section{Resumen}

La declaración de contingencia del COVID-19 ocurrida en Marzo del presente año trajo consigo cambios en los hábitos de compra de los consumidores ya que el cierre de comercios, tiendas departamentales y la reducción de la atención presencial al público en bancos y otros servicios provocó que aumentará el número de personas que hacen sus compras a través del comercio electrónico. El presente estudio analiza como el COVID-19 tiene un impacto en la incidencia de compra en línea de los jóvenes Millenials. Se aplicó una encuesta en línea a 187 personas del Noreste de Nuevo León pertenecientes a la mencionada generación, en un rango de edad de los 25 a 35 años. Los resultados obtenidos en este estudio muestran marcadas diferencias entre los hábitos de compra en línea entre hombres y mujeres, quienes realizan transacciones en aplicaciones de servicios bancarios y páginas de tiendas para realizar compras de diversos productos, esto con el fin de salvaguardar su salud.

Palabras Clave: comercio electrónico, COVID-19, generación Millennial 


\section{Introducción}

En la actualidad es común realizar transacciones a través del comercio electrónico, pero ¿Qué es el comercio electrónico? Este surge en 1948 pero no fue hasta los años setentas que la industria bancaria empezó a utilizarlo, y con la llegada de las tecnología de la información en los años 90's empezó a crecer su uso en diferentes industrias, lo cual trajo una ventaja competitiva con respecto a otros competidores en dos aspectos principalmente, tomar decisiones oportunamente y con estas, prestar mejor servicio a los consumidores, el comercio electrónico le brinda a las empresas la oportunidad de crear productos nuevos, reducir costos, nuevos canales de distribución y la apertura de nuevos mercados (Llanes, 1999; Fernández, Sánchez, Jiménez y Hernández, 2015).

El comercio electrónico ha cambiado la forma en que las personas cubren sus necesidades realizando sus compras habituales no solo de forma presencial sino a través del comercio electrónico, en esta era digital ya no sólo se compra en una tienda cercana a nuestro hogar o supermercados, hoy en día se realizan transacciones en línea de productos tales como comida, ropa y electrónica para que lleguen a nuestro hogar sin tener que salir de casa (Sigmond, 2018).

Es en Marzo del año 2020 que se marca un antes y un después en la vida cotidiana, desde que se declaró la pandemia por el COVID-19, esta no solo representa una amenaza grave para la salud, sino que rápidamente también impactó otras áreas, la economía del país se vio afectada con los cierres de negocios y por ende a los consumidores el distanciamiento social, provoca un cambio en sus hábitos ya que son apartados de sus interacciones sociales tales como reuniones, o cualquier tipo de interacción física, lo que incluye ir a centros comerciales, tiendas y la forma de adquirir productos (Campbell, Inman, Kirmani and Price, 2020).

El objetivo de este estudio es analizar como el comercio electrónico ha tenido un impacto con la declaración de la contingencia por el COVID-19 entre hombres y mujeres pertenecientes a la generación Millennial.

\section{2.- Revisión de Literatura}

\subsection{Comercio Electrónico}

Actualmente, escuchar el término comercio electrónico es algo común, la OECD (2011) lo define como la compra o venta productos y servicios a través de aplicaciones en la web tales como: extranet, EDI por Internet, entre otras. Por su parte, Nemat (2011) lo considera como el comercio electrónico es cualquier compra llevada a cabo a través de la web y cuyos pagos se hacen de forma electrónica. Con base a los anterior, el comercio electrónico son los servicios ofrecidos en Internet que permiten el acceso a bienes y servicios como lo son, banca en línea, entretenimiento, turismo, tiendas departamentales, entre otras y que está libre de restricciones temporales y territoriales (Yoon y Occena, 2015).

Para Gutiérrez (2015) el comercio electrónico es aquel a través del cual se hacen diferentes transacciones oferentes y un consumidor efectúa la compra de productos, servicios, transacciones en la banca electrónica y actividades comerciales como compra, de tal forma que al financiar productos o servicios se realice a través de redes abiertas, es decir, de la World Wide Web.

Como lo plantea Sigmond (2018) el comercio electrónico son las transacciones de compra y venta de bienes y/o servicios a través de medios electrónicos, este tiene tres categorías las cuales son: B2B (Business to Business) que es el comercio electrónico que se lleva a cabo entre empresas, B2C (Business to Customer) comercio entre empresas y consumidores, $\mathrm{C} 2 \mathrm{C}$ (Customer to Customer) se realiza entre consumidores. Esta investigación está centrada en el comercio entre empresa y consumidores finales, específicamente en la Generación Millenials.

Según datos de Statista (2010) el valor del comercio electrónico en México alcanzó los 14,500 millones de dólares en 2019 en donde gran porcentaje de las compras son hechas por personas entre los 20 y 36 años. Según la Asociación Mexicana de Venta Online, AMVO (2020) durante el año 2020 muestra que cinco de cada diez empresas en México duplicaron sus ventas online y dos de cada diez aumentaron en un $300 \%$ sus ventas mediante el comercio electrónico y el uso de aplicaciones de ventas en línea subió al 90\%.

\subsection{Generación Millenials}

Desde hace algunos años, la generación Millenials ha sido estudiada en diversas investigaciones, y aunque no hay un cien por ciento de exactitud en el rango de edad de las personas, la mayoría de los autores establecen que son los nacidos entre 1981 y 2000 (DeVaney, 2015). Se les conoce también como generación Y, generación IPod, generación global o nativos digitales, esto debido a que son la primera generación que desde su nacimiento están inmersos en un entono digital, lo que les ha condicionado la forma en que trabajan, como se relacionan con los demás, sus valores y las decisiones de compra 
(Leung, 2003; Bennet; Maton \& Kervin, 2008; Wesner \& Miller, 2008)

Para Duffett (2015) la generación Millenials se ha convertido en el primordial segmento de consumidores a nivel mundial. Según datos del INEGI (2020) en México hay 37.7 millones de Millenials que equivale al 30\% de la población total del país, estos jóvenes tienen características singulares en sus hábitos y forma de ver la vida, son tolerantes, buscan un equilibrio entre su vida personal y el trabajo, les gusta colaborar en equipo, son optimistas, muy individualistas, les gusta la tecnología, tienen un fuerte sentido de identidad, son modernos, les gusta emprender, son consumistas y materialistas (Hyllegard, Yan, Ogle \& Attmann, 2010; Burstein, 2013). Los jóvenes de la generación Millenials están muy expuestos a la tecnología, para ellos usar las redes sociales y estar conectados es muy significativo, tienen capacidades multiplataforma y son multitarea, para ellos es importantes socializar, en una generación empoderada, no se dejan influenciar por la publicidad tradicional, son críticos y exigentes (Ruiz, 2017).

\subsection{Comercio electrónico y la generación Millenials}

Según reportes de Statistic (2020) el 60\% de los Millenials compran a través del comercio electrónico, factores como la confianza, ser parte de una comunidad y poder comprar las 24 horas, la facilidad de pago en aplicaciones y la entrega a domicilio influye en el comportamiento de compra de estos jóvenes. Siguiendo con lo anterior, los Millenials prefieren hacer compras en empresas que tengan una tienda virtual o aplicaciones en línea, gran parte de ellos compran sus productos en grupos de las redes sociales, esto porque no requieren una computadora y lo hacen a través de distintos dispositivos electrónicos (Anaya, Castro y González, 2020).

El comercio electrónico ha evolucionado a lo largo del tiempo, la introducción de la redes sociales, y aplicaciones permite una combinación de medios sociales y comercio que ha impactado a los jóvenes de la generación Millenials, esto porque permite que los compradores interactúen en actividades de compra social, búsqueda de productos, socializar e intercambiar información a través de las páginas de negocios tradicionales, redes sociales como Facebook e Instagram, webs dedicadas a la venta de alimentos e insumos, servicios financieros, viajes y funciones de compras a través de sitios como Amazon (Molinillo, Liébana y Anaya, 2016; Shamugan, Sun; Amidi, Khani \& Khani, F., 2018).

\subsection{Comercio electrónico entre hombres y mujeres}

En una encuesta realizada por la Comisión Europea (2017) el porcentaje de jóvenes pertenecientes a la generación Millennial es del 86\%, mientras que en México representan el 60\% según datos de la OECD (2019).

Con respecto al género, existe una reducida brecha entre hombres y mujeres, según datos de la OCDE (2018) el 58\% de los hombres son más propensos a realizar compras a través del comercio electrónico contra el $56 \%$ de las mujeres, una diferencia poco significativa, por otro lado, en México el hombre promedio tenía el $45 \%$ más probabilidades de hacer compras en línea que la mujer promedio. La Asociación Mexicana de Internet (2019) realizó un estudio en México en donde los Millennial representan el 40\% de los compradores en línea de los cuales, el 51\% son hombres y el 49\% son mujeres. Los productos más comprados en el 2019 fueron transporte $65 \%$, 47\% en comida rápida, turismo un 45\%, hospedaje el $41 \%$, ropa y accesorios un $34 \%$, super y despensa $23 \%$.

En comparación con las generaciones anteriores a los Millenials estos tienen un mayor interés en el uso de Internet para diferentes aspectos de su vida cotidiana tales como: educación, redes sociales, entretenimiento y compras en línea (Castaño, Duart y Sancho, 2014). Por su parte Hargittai y Shaw (2015) afirman que existen diferencias de género entre los Millenials en el uso de internet, Los hombres prefieren contenido relacionado con las finanzas, política, deportes, video juegos y humor, en cambio, las mujeres prefieren contenidos sobre la familia, el hogar y la religión.

Después de hacer un estudio entre jóvenes Millenials en Italia, Nadeem, Andreini y Laukkanen (2015) comprobaron que las mujeres están más motivadas y hacen más compras a través del comercio electrónico que los hombres, quienes se muestran más cautelosos. Por otro lado, Petrovic y Pavlovic (2016) realizaron un estudio en Serbia, Macedonia y Bulgaria el cual arrojó resultados similares, las mujeres usan más el comercio electrónico, principalmente la banca en línea y en menos medida la compra y venta de productos y servicios turísticos.

\subsection{El impacto del COVID-19 en el comercio electrónico}

Desde principios de este año, hablar de coronavirus es algo recurrente en pláticas de amigos y familia, ¿Qué es esta enfermedad y porque entró de repente en la vida de todos los que habitamos este mundo? Según la Organización Mundial de la Salud (2010) una pandemia sucede cuando se propaga una enfermedad a nivel mundial, en marzo de 2020 se hace la declaratoria de pandemia de COVID-19. El coronavirus es un virus que puede causar enfermedades en humanos y animales con características que van desde un resfriado común hasta llegar a dificultades para respirar, síntomas que se 
presentan en los síndromes de MERS (Síndrome respiratorio de Medio Oriente) y SRAS (Síndrome Respiratorio Agudo Severo) acompañado de fiebre alta, dolor de cabeza, tos seca dolor y dolor de garganta.

Las medidas adoptadas para reducir el impacto del COVID-19 han afectado la oferta, la demanda y las operaciones de distintos factores, especialmente el de los minoristas que incluye la venta de productos tales como: vehículos motorizados, alimentos, abarrotes, ropa, entre otros; lo que ha provocado que estas empresas desarrollen formas alternas a las tradicionales para aumentar las ventas y la respuesta a ellos es el comercio electrónico (OCDE, 2020)

Para Huang y Sengupta (2020) desde la aparición del COVID-19 esté ha ejercido en los consumidores una influencia en que productos comprar y cuáles no y la forma de adquirir estos productos, pues ante las restricciones para salir a los supermercados y tiendas departamentales la opción más viable es realizar compras a través del comercio electrónico.

H1. La contingencia por COVID-19 incrementó el uso del comercio electrónico en las personas de la generación Millenials.

\section{3.- Metodología}

Esta investigación presenta un enfoque cuantitativo, no experimental y transversal.

La recolección de datos se realizó en un solo momento por medio de la aplicación de encuestas. El uso de encuestas en la metodología de la investigación permite extender las deducciones de una muestra a una determinada población lo cual permite inferir conclusiones acerca de los rasgos, conductas y cualidades de la población en su conjunto (Babbie,1990).

La realización de esta investigación toma como base una encuesta de diez reactivos como instrumento de evaluación, la cual se diseñó para conocer el comportamiento en los hábitos de compra en línea en cuanto a la frecuencia con la que realizan compras, las plataformas que utilizan, los tipos de transacciones y los montos de los gastos que la generación Millenials realizó antes de la pandemia y durante la pandemia de COVID-19 y determinar si se incrementó el uso del comercio electrónico entre las personas de dicha generación. La encuesta también nos permitió conocer las diferencias que hay entre los hombres y mujeres en su comportamiento al realizar compras en el comercio electrónico. La encuesta se aplicó a 187 personas de Nuevo León, de la generación Millenials cuyas edades oscilaron entre los 20 y 35 años durante los meses de octubre y noviembre del presente año (2020) vía electrónica, utilizando la plataforma Forms.

\section{4.- Resultados}

Para el análisis de los datos recolectados se utilizó Excel de Microsoft Office 365, los cuáles se presentan a continuación.

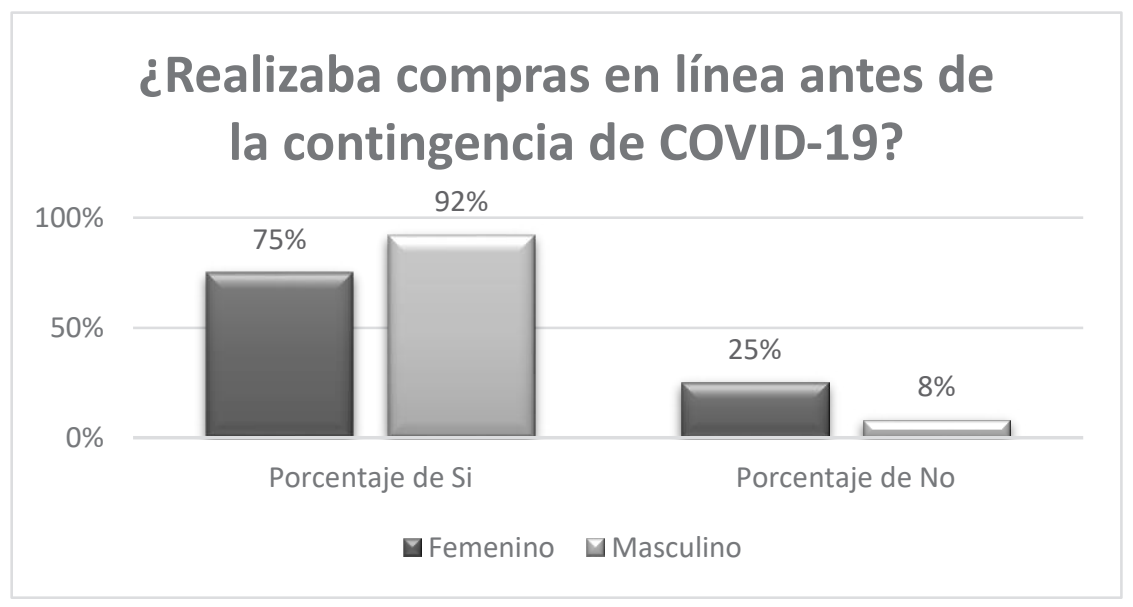

Figura 1. Compras en línea antes de la contingencia.

La figura 1 nos muestra que un mayor porcentaje de hombres con respecto a las mujeres ya realizaba compras en línea antes del COVID-19. El 75\% de las mujeres y el 92\% de los hombres de la generación de acuerdo con los resultados obtenidos. Se observa que un mayor porcentaje de hombres ya realizaba compras en línea antes de la contingencia lo que coincide con lo expresado por la OCDE (2018). 


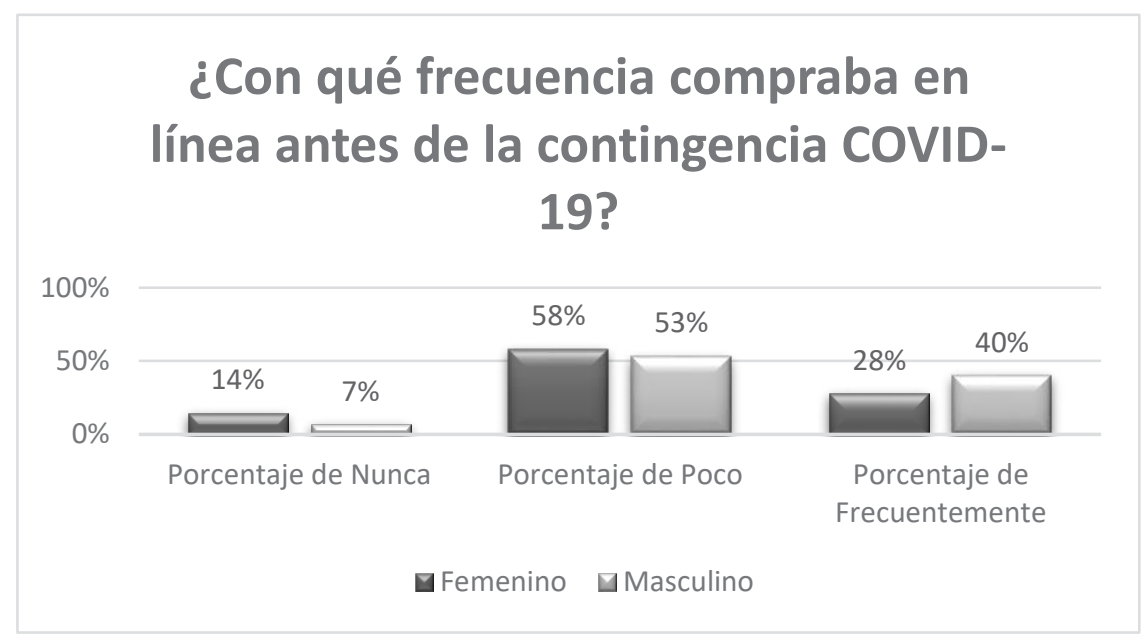

Figura 2. Frecuencia de compras antes de la contingencia

En la figura 2 podemos ver la frecuencia con la que se compraba en línea antes de la contingencia COVID-19. Los resultados muestran que el 14\% de las mujeres nunca realizó compras, el 58\% realizó compras con poca frecuencia y el $28 \%$ frecuentemente. En comparación, el 7\% de los hombres nunca realizó compras, el 53\% realizó pocas compras y el $40 \%$ realizó compras frecuentemente. Se observa como los hombres antes de la pandemia realizaban compras con mayor frecuencia que las mujeres, $40 \%$ de los hombres y un 28\% las mujeres. Los hombres de la generación Millenials presentan un mayor comportamiento de compras en línea.

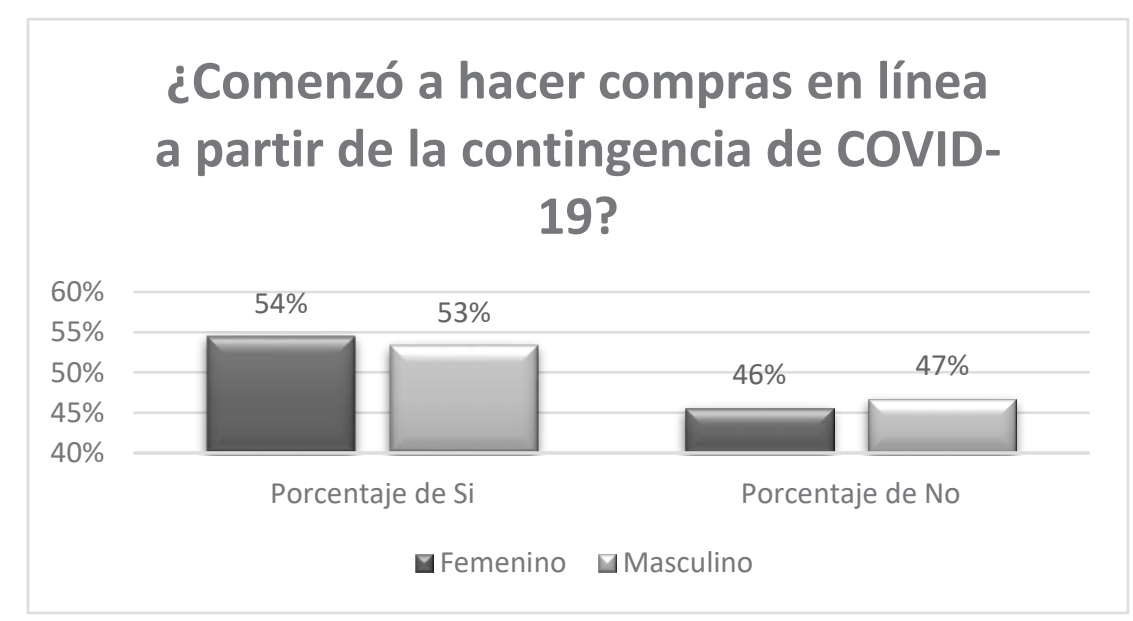

Figura 3. Compras en línea después de la contingencia.

La figura 3 nos muestra los resultados obtenidos en cuanto a las compras en línea a partir de la contingencia COVID-19. El $54 \%$ de las mujeres que no realizaban compras en internet, comenzó a realizarlas y el $53 \%$ de los hombres que no realizaban compras en internet comenzó a realizarlas. Los resultados muestran que se ha incrementado la compra en línea tanto en los hombres cómo en las mujeres. 


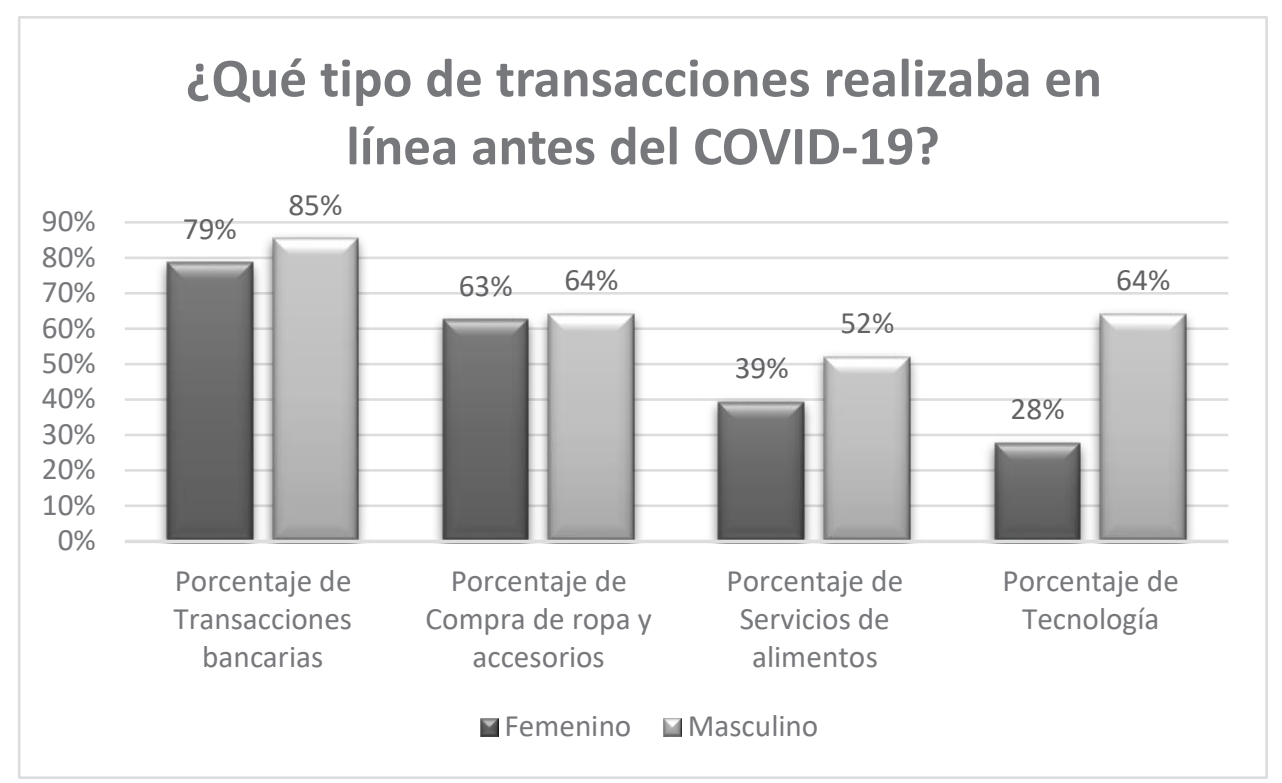

Figura 4. Tipo de transacciones en línea antes del COVID-19

En la figura 4 presentan los tipos de transacciones que realizaban en línea las personas de la generación Millenials antes del COVID-19. El 79\% de las mujeres realizaba transacciones bancarias, el 63\% compra de ropa y accesorios, 39\% solicitaba servicios de alimentos y el $28 \%$ compraba tecnología. Las transacciones realizadas por los hombres son el $85 \%$ transacciones bancarias, el 64\% ropa y accesorios, el 52\% solicitaba servicios de alimentos y el 64\% compraba tecnología. Los resultados anteriores nos permiten determinar que las transacciones bancarias son las que más se realizaban tanto por hombres como por mujeres. En cuanto a la compra de ropa y accesorios, el porcentaje de compra era muy similar. Pero existe una gran diferencia en cuanto a la compra de tecnología entre hombres y mujeres, mientras que el $64 \%$ de los hombres compraba tecnología, solamente el $28 \%$ de las mujeres la adquiría antes de la pandemia.

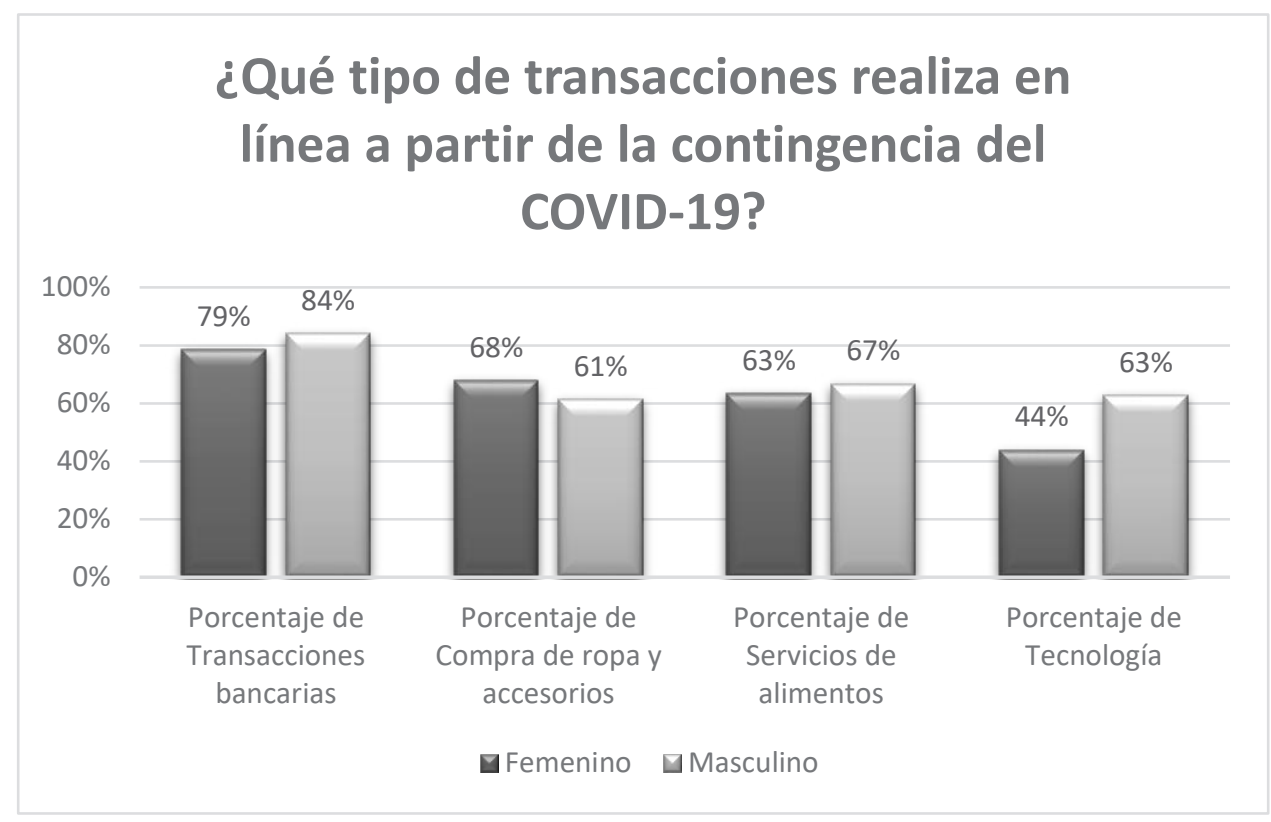

Figura 5. Tipo de transacciones en línea a partir COVID-19 
El tipo de transacciones bancarias que realiza en línea la generación Millenials a partir de la contingencia COVID-19 de acuerdo con los resultados mostrados en la figura 5 son los siguientes: el $79 \%$ de las mujeres realiza transacciones bancarias, el 68\% compra ropa y accesorios, el 63\% solicita servicios de alimentos, y el 44\% compra tecnología. En el caso de los hombres el $84 \%$ de lleva a cabo transacciones bancarias, el 61\% compra ropa y accesorios, el $67 \%$ solicita servicios de alimentos y el 63\% compra tecnología. La figura 5 nos muestra como cambiaron algunos de los hábitos de compra en línea a partir de la pandemia. Mientras que el porcentaje de transacciones bancarias y la compra de ropa y accesorios se mantiene casi igual tanto en hombres como en mujeres, antes y durante la pandemia, se observa que la solicitud de servicio de alimentos se incrementó. En las mujeres del 39\% al 63\% y en los hombres del 52\% al 67\%. En cuanto a la compra de tecnología se presenta un incremento representativo en las mujeres del $28 \%$ al $44 \%$. Mientras que en el caso de los hombres la compra de tecnología se mantiene en porcentaje similar.

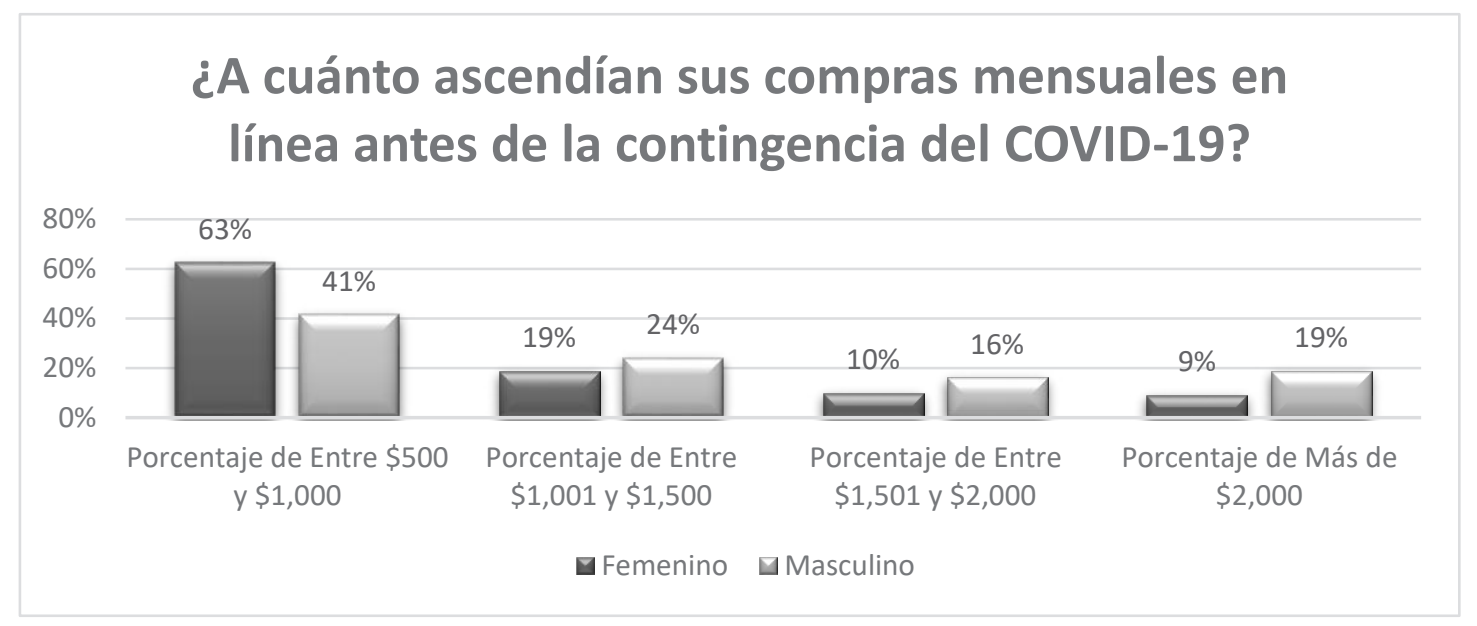

Figura 6. Montos de compra mensuales antes del COVID-19

En la figura 6 podemos observar a cuánto ascendían las compras mensuales en línea antes de la contingencia COVID-19. El $63 \%$ de las mujeres realizaban compras comprendidas entre $\$ 500$ y $\$ 1000$ pesos, el $19 \%$ entre $\$ 1001$ y $\$ 1500$, el $10 \%$ entre $\$ 1501$ y $\$ 2000$ y el $9 \%$ más de $\$ 2000$ mensuales. En el caso de los hombres el $41 \%$ entre $\$ 500$ y $\$ 1000$ pesos, el $24 \%$ entre $\$ 1001$ y $\$ 1500$, el 16\% entre \$1501 y \$2000 y el 19\% más de \$2000 mensuales. En los resultados podemos observar cómo los hombres gastaban más en las compras mensuales en comparación con las mujeres. El 19\% de los hombres gastan más de \$2000 pesos mensuales y solo el 9\% de las mujeres gastaba más de \$2000 mensuales. Podemos observar además, que el $63 \%$ de las mujeres gasta entre $\$ 500$ y $\$ 1000$ pesos mensuales en comparación con el $41 \%$ de los hombres. Las mujeres de la generación Millenials gastaban menos que los hombres en sus compras hechas en línea, antes de la pandemia.

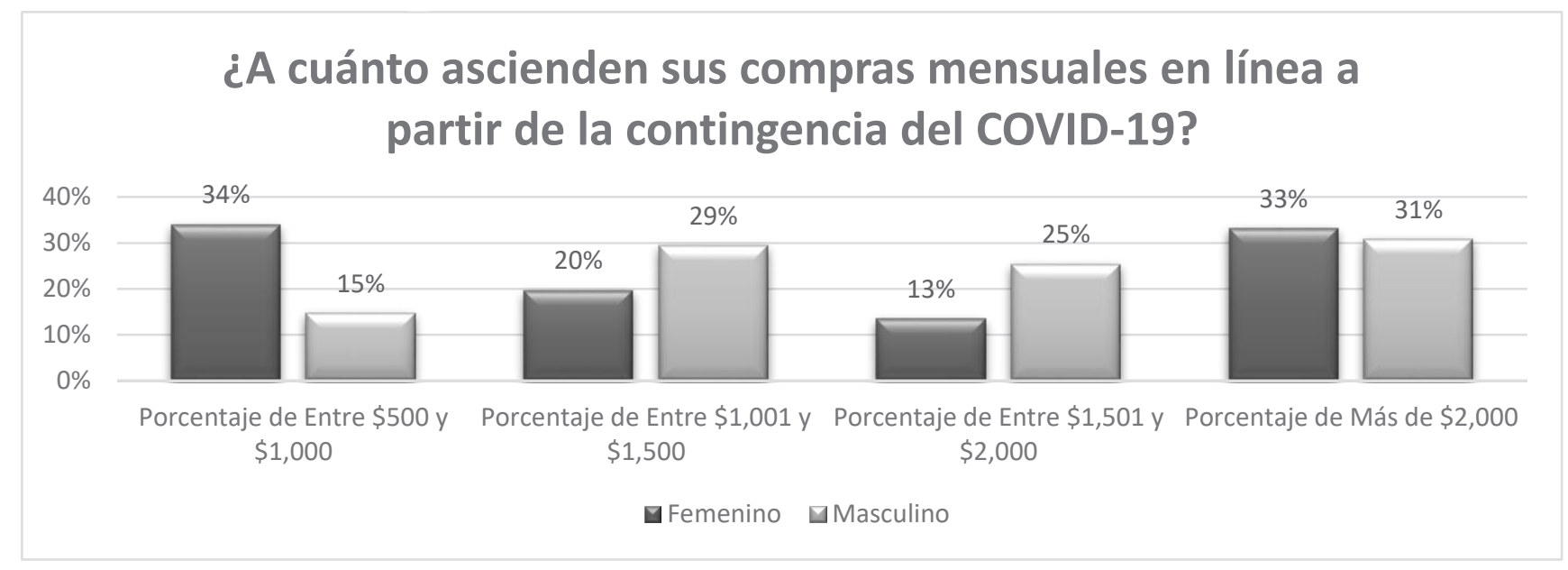

Figura 7. Montos de compra mensuales a partir del COVID-19 
La figura 7 muestra a cuánto ascienden las compras mensuales en líneas realizadas por las personas de la generación Millenials a partir de la contingencia COVID-19. El 34\% de las mujeres realizan compras comprendidas entre \$500 y $\$ 1000$ pesos, el $20 \%$ entre $\$ 1001$ y $\$ 1500$, el 13\% entre $\$ 1501$ y $\$ 2000$ y el 33\% más de $\$ 2000$ mensuales. Los resultados obtenidos para los hombres son: el 15\% realiza compras entre $\$ 500$ y $\$ 1000$ pesos, el 29\% entre $\$ 1001$ y $\$ 1500$, el $25 \%$ entre \$1501 y \$2000 y el 31\% más de \$2000 mensuales.

Si comparamos los resultados presentados en las figuras 6 y 7 se observa cómo se incrementan los porcentajes de compras en más de $\$ 2000$ pesos mensuales para las mujeres, se incrementa de $9 \%$ a 33\% y en los hombres sube de $19 \%$ a $31 \%$. Se aprecia también un aumento en las compras entre \$1501 y \$2000 pesos, en las mujeres del 10\% al 13\% y en los hombres del $15 \%$ al $25 \%$. Hay una disminución muy significativa en las compras entre $\$ 500$ y $\$ 1000$ pesos, en las mujeres disminuye de $63 \%$ a $34 \%$ y en los hombres disminuye de $41 \%$ a $15 \%$. Por los resultados anteriores podemos darnos cuenta de que la influencia del COVID-19 incrementó significativamente el uso del comercio electrónico en la generación Millenials tanto en el sexo femenino como en el masculino.

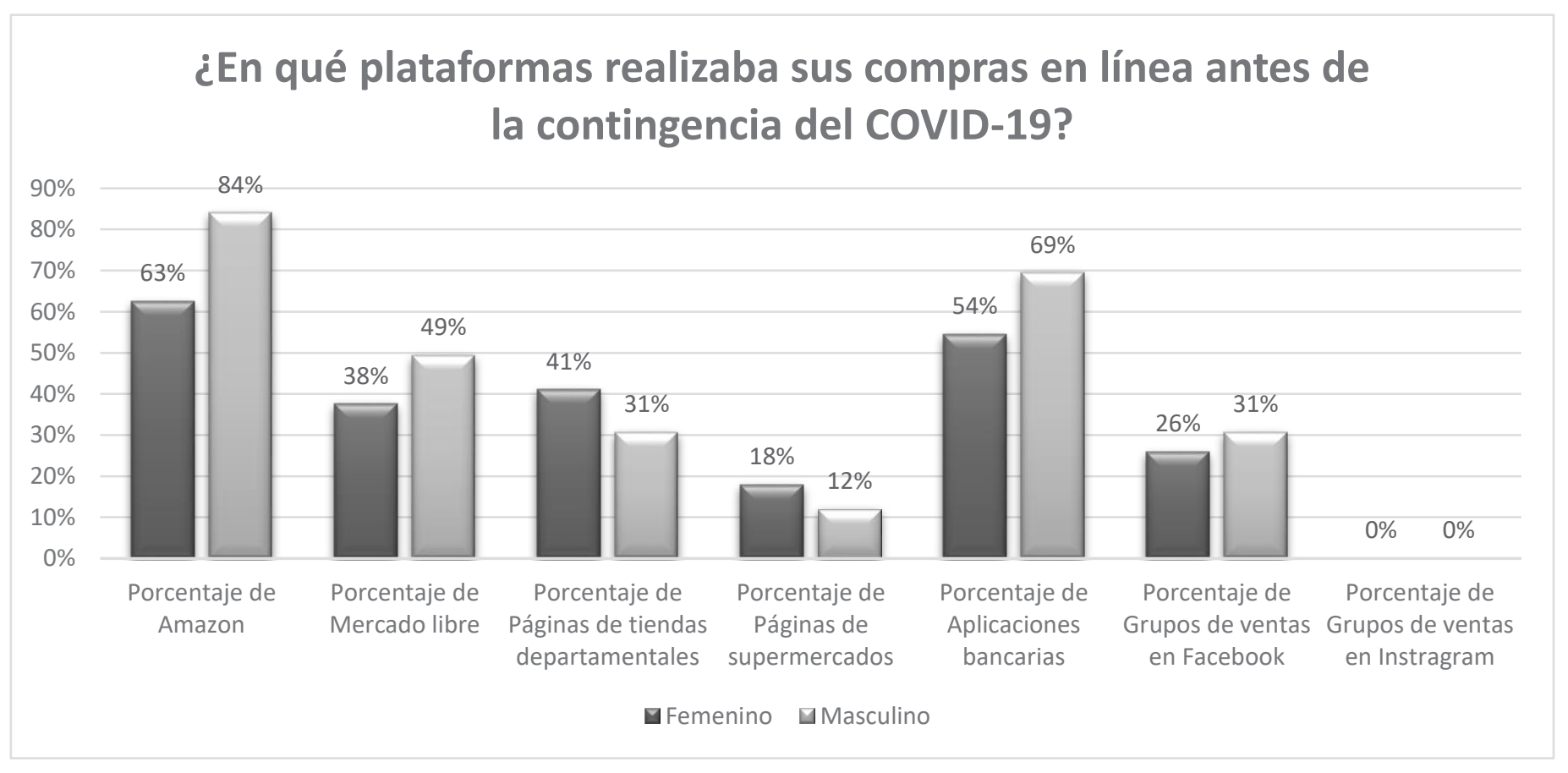

Figura 8. Plataformas utilizadas para compras antes del COVID-19

E1 uso de las plataformas antes de la contingencia COVID-19 se muestra en la figura 8. Las mujeres utilizaban en un 63\% Amazon, 38\% Mercado Libre, 41\% páginas de tiendas departamentales, 18\% páginas de supermercados, 54\% aplicaciones bancarias, 26\% grupos de ventas en Facebook y 0\% ventas en Instragram. El uso de las plataformas antes del COVID-19 por los hombres fue un $84 \%$ Amazon, 49\% Mercado Libre, 31\% páginas de tiendas departamentales, $12 \%$ páginas de supermercados, 69\% aplicaciones bancarias, 31\% grupos de ventas en Facebook y 0\% ventas en Instragram. 


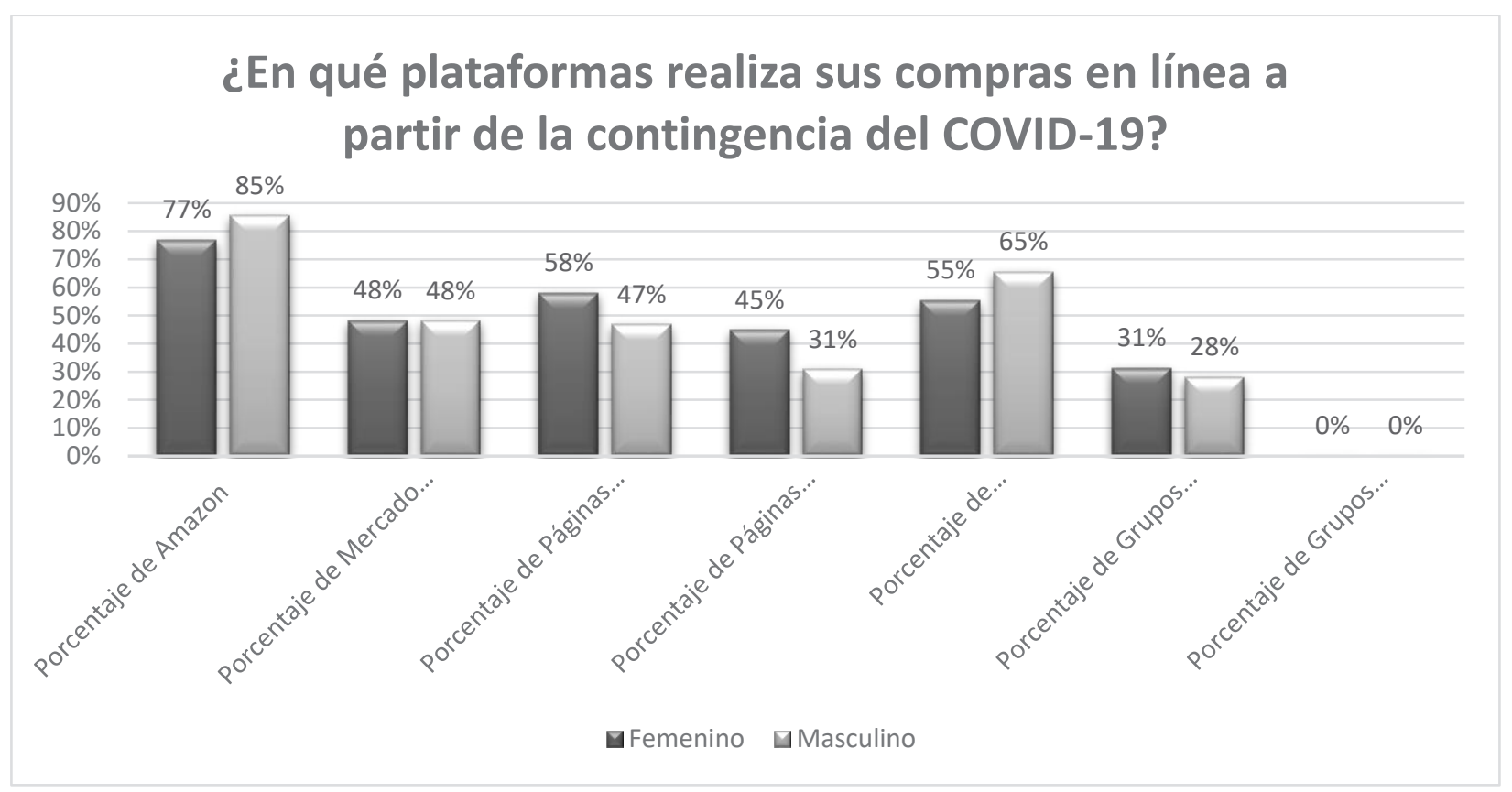

Figura 9. Plataformas utilizadas para compras durante COVID-19

En la figura 9 se muestra en que plataformas realizan los hombres y mujeres sus compras en línea a partir de la contingencia COVID-19. Las mujeres utilizan en un 77\% Amazon, 48\% Mercado Libre, 58\% páginas de tiendas departamentales, $45 \%$ páginas de supermercados, 55\% aplicaciones bancarias, $31 \%$ grupos de ventas en Facebook y $0 \%$ ventas en Instragram. El uso de las plataformas a partir del COVID-19 por los hombres es, un 85\% Amazon, 48\% Mercado Libre, $47 \%$ páginas de tiendas departamentales, $31 \%$ páginas de supermercados, $65 \%$ aplicaciones bancarias, $28 \%$ grupos de ventas en Facebook y 0\% ventas en Instragram.

Si comparamos el uso de las plataformas antes de la contingencia y durante la contingencia, nos damos cuenta que en el caso de las mujeres se incrementó considerablemente el uso de Amazón del 63\% al 77\% y de Mercado Libre del 38\% a $48 \%$, en el caso de los hombres, en cambio, es mínimo en ambas plataformas. En cuanto a las plataformas de las tiendas departamentales hay un aumento en ambos sexos. En las mujeres hay un incremento del $41 \%$ al $58 \%$ y en los hombres del $31 \%$ al $47 \%$. Así mismo se muestra incremento muy significativo en el uso de las plataformas de páginas de supermercado, en mujeres del 18\% al $45 \%$ y en los hombres del $12 \%$ al $31 \%$. El uso de las plataformas bancarias es muy similar en ambos casos. Se observa un ligero incremento en las compras en Facebook por parte de las mujeres. Y no se realizan compras por Instagram ni antes ni durante la contingencia COVID-19.

Los resultados muestran un cambio por parte de la generación Millenials en el uso de las plataformas donde se realizan las compras en líneas, observándose claramente un incremento en el uso de estas a partir de la pandemia. 


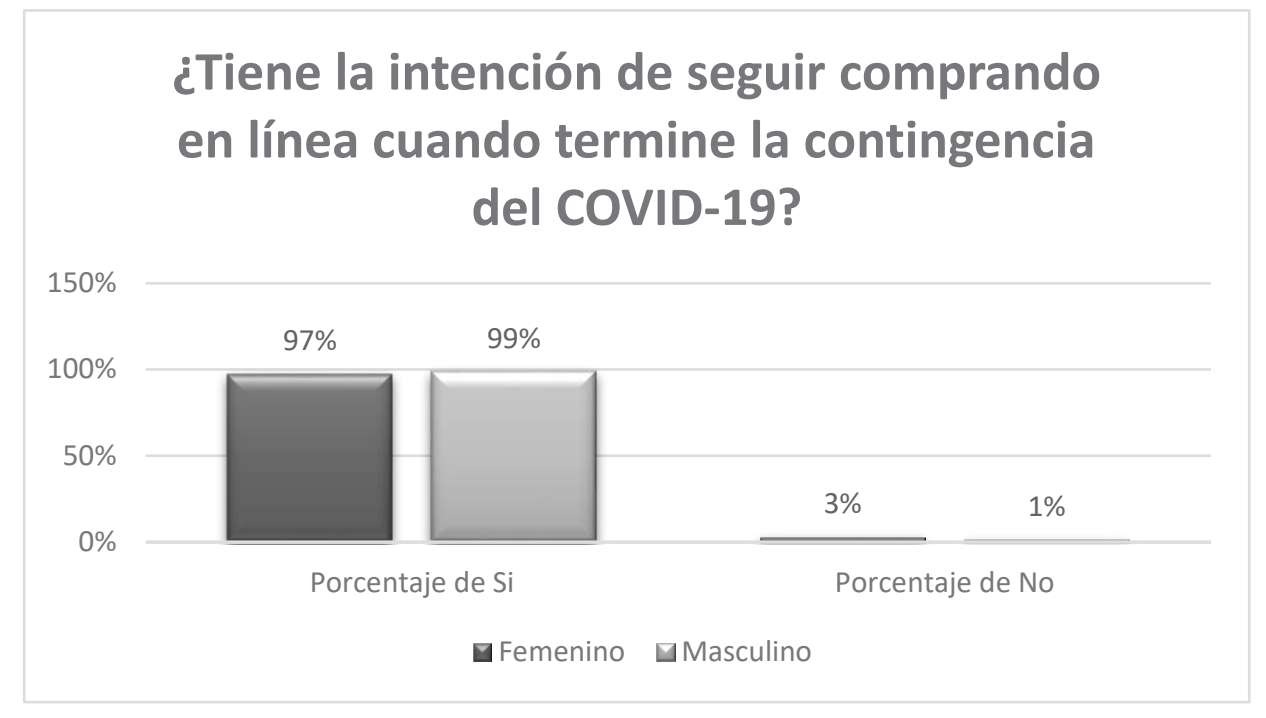

Figura 10. Intención de compras después del COVID-19

En la figura 10 se nos muestra que el $97 \%$ de las mujeres de la generación Millenials tiene la intención de seguir comprando en línea cuando termine la contingencia del COVID-19 contra un 3\% que respondieron que no. Y en el caso de los hombres el 99\% expresa tener la intención de seguir comprando en línea y un 1\% expresa que no tiene la intención de seguir comprando en línea cuando pase la contingencia.

Los resultados arrojados por la presente investigación permiten comprobar que la contingencia por COVID-19 incrementó el uso del comercio electrónico en las personas de la generación Millenials, por lo que la hipótesis (H1) planteada es aceptada.

\section{5.- Conclusiones.}

Debido a las restricciones impuestas por los organismos de salud en el país, con el fin de controlar y evitar los contagios por COVID-19, los consumidores al no poder asistir a los supermercados, tiendas departamentales e instituciones bancarias han tomado la alternativa de hacer sus compras y transacciones en línea, utilizando las páginas que las mismas tiendas y bancos ofrecen, esto con el afán de salvaguardar su salud.

Así mismo, los resultados de esta investigación nos muestran marcadas diferencias entre los hábitos de compra en línea y la preferencia en el uso de ciertas plataformas que existen entre los hombres y las mujeres de la generación en estudio.

La contribución de esta investigación a la literatura es que los resultados encontrados se obtuvieron de una generación específica en la cual se ha observado el cambio en su comportamiento con respecto a las compras en línea y cómo debido a la contingencia estas se han visto incrementadas.

Es importante mencionar que la muestra utilizada para el estudio se tomó específicamente de jóvenes Millenials que viven en el noreste de México, lo cual es una limitante del mismo, por lo que los resultados de este estudio no se pueden generalizar. Se recomienda para futuros estudios tomar en cuenta estas limitaciones.

\section{REFERENCIAS}

Anaya, R., Castro, J. M., and González, E. (2020). Preferencias del consumidor Millenials respecto al diseño de webs de social commerce. Revissta Brasileira de Gestao de Negocios, 22(1), 123-139.

Asosiación Mexicana de Internet MX. (2019). Estudio sobre el comercio eletrónico en México 2019. estadística Digital, 13(1), 11-16.

Babbie, E. (1990). Survey Research Methods (Primera edición ed.). Wadsworth Publishing Company.

Bennet, S., Maton, K., and Kervin, L. (2008). The "digital natives" debate a critical review of the evidenca. British Journal of Educational Tecnology, 39(5), 775-786.

Burstein, D. (2013). Fast Future: How the Milenial Generation is Shpaing Our World. Boston: Beacon Press.

Campbell, M., Inman, J., Kirmani, A., and Price, L. (2020). In times of trouble: a framework for understanding consumer's responses to threats. Joournal of consumer, 47(3), 311-326. 
Castaño, J., Duart, J., and Sancho, T. (2014). The Internet in face to face higer education: can interctive improve academic achievemnt. Brisitsh Journal of Educational Technology, 45(1), 149-159.

DeVaney, S. A. (2015). Understanding the Millennial Generation. Journal of Financial Service Professionals, 69(6), 11-14.

Duffett, R. G. (2015). Facebook advertising's influence on intention topurchase and purchase amongst Milenials. Internet Research, 25(4), 498-526.

European Commission. (2017). Final report on the e-commerce sector inquiry. European Commision, 5-35.

Fernandez, A., Sánchez, M., Jiménez, H., y Hernández, R. (2015). La importancia de la innovación en el Comercio Electrónico. Universia Business Review, 1(47), 106-125.

Hargittai, E., and Shaw, A. (2015). Mind the skills gap: the role of Internet know how and gender in differentiated contributions to Wikipedia. Information, Communication \& Society, 18(4), 424-442.

Huang, Y., and Sengupta, J. (2020). The influence of disease cues on preference fot typical versus atypical products. Journal of consumer research, 47(3), 393-411.

Hyllegard, K., Yan, R., Ogle, J., and Attmann, J. (2010). The influence of gender, social cause, charitable support, and message appeal on Gen Y's responses to cause-related marketing. Journal of Marketing Management, 27(1-2), 100-123.

Illanez, P. (1999). El sistema empresa. Una visión integral de la administración. Santiago de Chile: Leed impresores.

Infochanel. (2020). Infochanel. Obtenido de https:/www.infochannel.info/amvo-covid-19-favorece-ecommerce-en-mexico

Instituto Nacional de Estadística y Geografía. (08 de Diciembre de 2020). INEGI. Obtenido de https://www.inegi.org.mx/

Leung, L. (2003). impacts of net-generation attributes, seductive propierties of the internet, and gratificationsobtained on internet use. Telematics and infomatics, 20(2), 107-129. ternet_use

Molinillo, S., Liébana, F., and Anaya, R. (2018). A Social Commerce Intention Model for Traditional, E-Commerce Sites. Journal of Theoretical and Applied Electrónic Commerce Research, 13(2), 80-93.

Nadeem, W., Andreini, D., Salo, J., and Laukkanen, T. (2015). Engaging consumers online through websites and social media: a gender study of Italian Generatión Y clothing consumers. International Journal of information Management, 35(4), 432-442.

Nemat, R. (2011). Taking a look different types of e-commerce. World Applied Programming, 1(1), $100-104$.

OCDE. (2018). Panorama del comercio electrónico, politicas, tendencias y modelos de negocio. OCDE, 35-76.

OECD. (2011). Guide to measuring the information society. OECD Publising, 1-22.

Infochanel. (2020). Infochanel. Obtenido de https:/www.infochannel.info/amvo-covid-19-favorece-ecommerce-en-mexico

Petrovic, Z., and Pavlovic, D. (2016). Students preferences with regards to the use of internet content: gender differences. The Antropologist, 24(2), 407-415.

Ruiz, J. (2017). Millenials y redes sociales: estrategias para una comunicación de marca. Miguel Hernández Communication Journal, 12(104), 347-367.

Shanmugam, M., Sun, S., Amidi, A., Khani, F., and Khani, F. (2016). The applications of social commerce constructs. International Journal of Infomation Management, 36(3), 425-432.

Sigmond, K. (2018). El comercio electrónico en los tratados de libre comercio de México IUS. Revista del Instituto de Ciencias Jurídicas de Puebla AC., 12(41), 359-377.

Statista. (28 de Noviembre de 2020). Statista. Obtenido de https://es.statista.com/estadisticas/632410/valor-de-mercado-del-comercioelectronico-

mexico/\#: :text=El\%20valor\%20de\%20mercado\%20del,d\%C3\%B3lares\%20en\%20el\%20pa\%C3\%ADs\%20latinoamericano

Wesner, M., and Miller,, T. (2008). Boomers and Millenials have much in common. Organizational Development, 26(3), 89-96.

Yoon, H., and Occeña, L. (2015). Influencing factors of trust consumer to consumer electronic commerce with gender and age. International Journal of Information Management, 35(3), 352-363. 